\title{
POLÍTICAS PÚBLICAS DE INNOVACIÓN TECNOLÓGICA EN LA INDUSTRIA
} PETROLERA VENEZOLANA ${ }^{1}$

\author{
PUBLIC POLICIES OF TECHNOLOGICAL INNOVATION IN THE OIL INDUSTRY VENEZUELAN \\ Johana Quintero $^{2}$, Judeira Batista ${ }^{3}$, Esther Pereira ${ }^{4} \&$ Ramiro Nova $^{5}$ \\ VIVECA, GITEMCA, PDVSA-Venezuela/Universidad Autónoma de Bucaramanga-Colombia
}

RECIBIDO: Agosto 16 de 2016

ACEPTADO: Noviembre 12 de 2016

\section{RESUMEN}

La investigación tuvo como propósito analizar las políticas públicas en innovación tecnológica en la Industria Petrolera Venezolana, donde el Estado es responsable de fortalecer esta tarea como parte integral del desarrollo, siendo un reto para el País poder implementar acciones que tengan impacto y beneficien directamente a la población. Se soportó bajo el paradigma positivista, que considera la investigación descriptiva, no experimental, transeccional y de campo. La población fue de 52 informantes a los cuales se les aplicó un cuestionario de 24 ítems. Luego de desarrollar el análisis estadístico sobre los datos obtenidos a través del instrumento, se tuvo como resultado, respecto a la variable Políticas Públicas de innovación tecnológica, que la misma se ubicó en nivel moderado según el baremo de interpretación diseñado, concluyendo que existe disposición para impulsar la innovación tecnológica interna, carencia de una planificación y organización de carácter estratégico basado en la aplicación de las Políticas Públicas que favorezcan el desarrollo científico y tecnológico así como, la consolidación de una estructura socio-institucional y legal para la innovación tecnológica desarrollada por el Estado venezolano.

Palabras clave: políticas públicas, innovación tecnológica, industria petrolera

\begin{abstract}
The purpose of the research was to analyze the public policies of technological innovation in the Venezuelan Oil Industry, where the State is responsible for strengthening this task as an integral part of development, being a challenge for the Country in implementing actions that have an impact and a direct benefit to the population. It was supported under the positivist paradigm, which considers a descriptive, non-experimental, trans sectional, field research. The population of the survey included 52 informants to whom a questionnaire of 24 items was applied. After developing the statistical analysis on the data obtained through this instrument, the result was, in relation to the variable Public Policies of technological innovation that it was located at a moderate level according to the scale of interpretation designed. Finally concluding that there is a promotion of Internal technological innovation, there is a lack of strategic planning and organization, based on the application of Public Policies that favor scientific and technological development, as well as the consolidation of a socio-institutional and legal structure for technological innovation developed by the State of Venezuela.
\end{abstract}

Keywords: public policies, technological innovation, oil industry

\section{Este artículo se puede referenciar}

Quintero, J., Baptista, J., Pereira E. \& Nova, R. (2017). Políticas públicas de innovación tecnológica en la industria petrolera venezolana. En Desarrollo Gerencial Revista de la Facultad de Ciencias Económicas Administrativas y Contables de la Universidad Simón Bolívar-Colombia, 9(1), 35-51.

\footnotetext{
${ }^{1}$ Proyecto derivado de la investigación intitulada "políticas públicas de innovación tecnológica en la industria petrolera venezolana.

${ }^{2}$ Doctora en ciencias gerenciales, coordinadora de Control y Gestión en Viveres D'Cándido (VIVECA) johanaquintero7@gmail.com.

3 Doctora en Ciencias Gerenciales, Consultora Empresarial Gerencia, Ingeniería, Tecnología y mercadeo (GITEMCA), judeirabatista@gmail.com.

${ }^{4}$ Doctora en Ciencias Mención Gerencia, Analista Financiero de Petróleos de Venezuela (PDVSA), estherpereira456@gmail.com.

${ }^{5}$ Doctor en Ciencias Mención Gerencia, Docente Investigador Universidad Autónoma de Bucaramanga ranoval@msn.com.
} 


\section{1.- INTRODUCCIÓN}

La humanidad ha venido experimentando profundas como aceleradas transformaciones en todos sus contextos, sean estos de corte político, social o económico. La obsolescencia así como las aceleradas transformaciones dan lugar a la búsqueda y descubrimiento de otros que den respuesta a las diversas exigencias de cambios planteados por el mundo globalizado, a tal razón, en el campo de la gerencia, las organizaciones están en la exploración continua de estrategias para permitir mantenerse en el tiempo, ser competitivas; en este sentido, se señala la innovación como un componente importante cobrando auge dentro de la práctica gerencial. En este sentido, Prieto, Emonet, García y González (2015) plantean que dichas transformaciones, a nivel empresarial, se hacen necesarias en el mundo adaptativo donde las organizaciones se encuentran sometidas a demandas diferentes, motivo por el cual las mismas deben modificarse, adecuarse para poder responder, permanecer y mejorar en el mercado, todo ello, orientado hacia un desarrollo organizacional eficaz.

Atendiendo a estas consideraciones, en el mundo estas prácticas gerenciales cobran fuerza cuando desde las asociaciones y organizaciones que existen emiten direccionamientos gerenciales que se convierten en políticas cuando siguen un rumbo determinado para favorecer intereses comunes, estos direccionamientos los denomina política Bourdieu (2005), cuando señala, la existencia del Estado, no solo requiere la presencia de las condiciones efectivas que la definen, como el territorio, una población, una autoridad entre otros, hace falta la política que le da rumbo hasta alcanzar un desarrollo socio productivo que le aporte a la nación, al país y al estado.

De igual forma, Cebrian (2003), explica que, cuando los Estados son concebidos como actores y no como espacios donde meramente se reflejan las demandas e intereses de los actores socioeconómicos, deben gozar de la suficiente autonomía para definir sus propios objetivos.

En este orden de ideas, es pertinente mencionar que (Podestá, 2006) señala que las políticas públicas, son instrumentos de trabajo, mediante el cual se pretende alcanzar desde el Estado, en forma sistemática y coherente, ciertos objetivos de interés para el bienestar de toda la sociedad. Ahora bien, cuando desde el Estado se plantean propuestas de mediano y largo plazo hacia la sociedad civil, existe la necesidad de ejecutar programas para cumplir los objetivos de interés social, y en este nivel las políticas públicas son un instrumento fundamental.

Las políticas públicas, por lo tanto, se reconocen como un proceso de aprendizaje colectivo para aumentar la capacidad de resolver problemas, influyendo de manera decisoria en la formulación y legitimación de la agenda pública a través de un proceso de interlocución y comunicación democrática entre sociedad y gobierno 
Se hace importante destacar que Venezuela, se encuentra en un momento caracterizado por profundas transformaciones en lo político, económico y social, a través de un proceso democrático guiado por la constitución venezolana. En este contexto, la importancia de las políticas públicas de innovación tecnológica en la industria petrolera venezolana, caracterizada porque el estado es responsables de fortalecer esta tarea siendo parte integral del desarrollo.

El desarrollo de la implementación tecnológica en procesos técnicos efectivos, ha generado beneficios económicos a las empresas y por ende a los países, esta noción unifica estrategias de avanzada, de conocimiento y organizacionales trayendo como efecto la implementación de procesos tecnológicos que reafirman la ventaja competitiva de las empresas en su área de acción. Así lo corroboran Enríquez, Salgado y Castonera (2013) cuando señalan que, las empresas para mejorar el desempeño y la competitividad de sus operaciones, requieren integrar las tecnologías a los procesos de producción,

La ventaja competitiva se soporta con la estrategia de cambios, impulsa la expansión de actividades que requieren a su vez transformaciones en la estructura de la empresa, modifica los estilos gerenciales y procedimientos entre otros. Esta circunstancia llama al desarrollo de nuevas capacidades o a efectuar el ajuste articulado de las existentes, con el fin de que estos cambios tecnológicos promuevan la supervivencia de las organizaciones frente a sus competidores, esta investigación plantea el análisis de la política pública de innovación tecnológica en la industria petrolera venezolana.

\section{Contexto del Problema}

Venezuela, al igual que la mayoría de los países latinoamericanos, intenta, desde el año 1989 desenvolverse según otro modelo económico como respuesta a los requerimientos que se desprenden de los nuevos giros que da la economía mundial y a los problemas que confronta su propia economía. Esto implica una transición que va desde el agotamiento del modelo rentista, posible gracias al petróleo, hacia una economía productiva, delineada según los cánones del mercado, en un contexto de apertura comercial. Este intento ocurre en medio de vastas y hondas transformaciones tecnológicas, las cuales implican nuevas e importantes exigencias para que tal modelo sea exitoso.

Los problemas en un país deben gestionarse de forma activa como parte del proceso de crecimiento, no se conseguirán mejoras hasta no producirse un aumento de los ingresos financieros aportando mayores recursos. Las políticas de innovación en general deben descansar sobre los principios políticos de consolidación de las instituciones y de gobernabilidad, integración de la sostenibilidad en todas las políticas sectoriales, mejora de los mercados y supresión de las subvenciones nocivas para la industria petrolera, refuerzo de los mecanismos nacionales e internacionales de gestión para la innovación, inversión en ciencia y tecnología. 
De la misma forma se afecta la composición de la demanda agregada al alterar el patrón de distribución del ingreso, y ocurren transformaciones en el sistema financiero, desarrollo de mercado de capitales, de capital de riesgo, entre otros, por otro lado, las nuevas tecnologías vienen acompañadas de un nuevo modelo organizativo y gerencial, basado en un concepto de máxima flexibilidad y agilidad de respuesta, mínimo inventario y cero defectos, cuya expresión organizativa e institucional más acabada se ubican en los países de Oriente..

Estas dos líneas de cambio convierten y se complementan de tal manera que han llegado a constituir un nuevo modelo de eficiencia productiva, aplicable en casi todas las ramas de la producción de bienes o servicios. Su incorporación en una rama tras otra, y va estableciendo niveles de productividad, calidad y capacidad de respuesta significativamente superiores a los que hasta ahora venían determinando la competitividad en los diferentes mercados

Un diagnóstico severo pone de nuevo sobre el tapete dificultades presentes para avanzar en el proceso de desarrollo, generación y distribución de riquezas y capacidades. Sin embargo, existen iniciativas y adelantos para desarrollar y consolidar un verdadero Sistema Nacional de Ciencia, Tecnología e Innovación: el marco legal y normativo y los servicios de financiamiento y de apoyo existentes, algunas de las instituciones actuales, la demanda de la industria petrolera, son adecuados para lograr la utilización de la oferta y las capacidades nacionales en ciencia y tecnología, todavía escasamente aprovechadas, en función del desarrollo de la productividad del sector empresarial y del país. Genatio (2004).

En este contexto, la importancia de las políticas públicas de innovación tecnológica en la industria petrolera, donde el estado es responsables de fortalecer esta tarea son parte integral del desarrollo, siendo un reto para el País poder implementar acciones que tengan impacto y beneficien directamente a la población. De la misma manera, no se ha prestado la debida atención a la innovación tecnológica, a pesar del potencial de este sector para contribuir a un incremento significativo del empleo y la producción, ganando cada vez más terreno y constituyéndose en un sector emergente dentro de la economía en la industria petrolera.

Sin embargo, una de las mayores dificultades se presenta en la industria petrolera nacional la cual concentra el mayor peso cuantificable en términos de generación de divisas para el país, constituyéndose la industria petrolera y energética estatal, la propulsora de toda la actividad económica nacional; en este contexto ocurre la aparición de las Empresas Mixtas como Filiales de PDVSA bajo el amparo de nuevos lineamientos corporativos sobre la reestructuración de la nueva PDVSA, manteniéndose en este modelo la herencia tecnológica de los antiguos convenios operativos, permitiendo así la subsistencia en cada una de las organizaciones creadas en el marco de este esquema de asociación, la cultura organizacional de los ahora socios minoritarios. 
De manera que la posibilidad del desarrollo innovador avanzado se mantiene supeditado al mayor o menor interés demostrado por los antiguos operadores empleando la conversión de procesos de adquisición/importación de las tecnologías en procesos de transferencia tecnológica necesaria para el manejo eficiente de estas estructuras, lo que reduce el desarrollo de capacidades de producción nacional para la industria petrolera, evidenciándose un consecuente aumento de la dependencia tecnológica trasnacional.

De igual manera no se evidencia una disposición para impulsar la innovación tecnológica interna, así como la carencia de una planificación y organización de carácter estratégico basado en la aplicación de las políticas públicas que favorezcan el desarrollo científico y tecnológico a partir de la consolidación de una estructura socio-institucional y legal para la innovación tecnológica desarrolladas por el Estado Venezolano.

En consecuencia, la presente investigación realiza un análisis de la política pública en innovación tecnológica para la industria petrolera, para cumplir con el objetivo fue necesario responder la siguiente interrogante Como es las políticas públicas en innovación tecnológica en la industria petrolera venezolana.

\section{2.- FUNDAMENTACION TEORICA}

Al respecto Salazar (2000), las define como el conjunto de sucesivas respuestas del Estado frente a situaciones consideradas socialmente como problemáticas. Las políticas públicas son las acciones y normativas que realiza el gobierno o el Estado para tratar de facilitar una determinada actividad (económica o no) y para mejorar su desempeño.

En uno de los elementos constitutivos de la noción de sistema político, se encuentra lo que se denomina como "políticas públicas". Se encuentran en el elemento institucional, llamado régimen político. Tal como señala Alcántara (1995), las mismas se consideran como determinados flujos del régimen político hacia la sociedad. Son concebidas como "productos del sistema político",

En otras palabras se puede decir, que las políticas públicas son las acciones de gobierno, es el gobierno en acción, que busca como dar respuestas a las diversas demandas de la sociedad, se pueden entender como uso estratégico de recursos para aliviar los problemas nacionales. Se trata pues, de extraer recursos de la sociedad para volver a colocarlos en ella.

En el mismo orden de ideas, en base a lo expresado por el mismo autor las políticas públicas son el conjunto de actividades de las instituciones de gobierno, actuando directamente o a través de agentes, y que van dirigidas a tener una influencia determinada sobre la vida de los ciudadanos, el autor señala que a esta definición genérica de políticas públicas hay que agregarle algunas consideraciones, tales como: 
Las políticas públicas deben ser consideradas como un proceso de toma de decisiones. Es decir por un conjunto de decisiones que se llevan a cabo a lo largo de un plazo de tiempo. Estas decisiones normalmente tienen una secuencia racional.

Una vez establecida esta definición de políticas públicas con sus debidas connotaciones, se tratan de abordar algunos de los instrumentos que utilizan las instituciones de gobierno que elaboran políticas públicas, que vienen a ser instrumentos de acción de las políticas públicas. Los instrumentos son: las normas jurídicas, los servicios, los recursos financieros y la persuasión.

Vásquez, M. (2004), define las políticas públicas se reconocen como un proceso de aprendizaje colectivo para aumentar la capacidad de resolver problemas, influyendo de manera decisoria en la formulación y legitimación de la agenda pública a través de un proceso de interlocución y comunicación democrática entre sociedad y gobierno.

Esta investigación define de manera amplia la innovación tecnológica y considera las etapas científicas, técnicas, comerciales y financieras, necesarias para el desarrollo y comercialización exitosa del nuevo o mejorado producto, proceso o servicio social. Para Martínez (2002), la innovación tecnológica, es como todo cambio significativo de una tecnología que logra imponerse en el mercado, o en términos más amplios, llega a emplearse en forma permanente por la sociedad, con el objetivo de contribuir a mejorar el desarrollo del sector productivo de bienes y servicios y a incrementar la calidad de vida mediante suministros de mejores productos a los consumidores.

Tal como lo señala este autor, la innovación tecnológica es un proceso donde la innovación, el desarrollo y la comercialización suman un todo de una forma muy simple se incluyen actividades de invención y explotación comercial, la invención considera actividades generadoras de ideas y determinación de factibilidad técnica y su trasformación en un producto de utilidad comercial que luego es adoptado por las personas y las empresas.

En términos de Oberto (2007), una invención o idea creativa no se convierte en innovación hasta que no se utiliza para cubrir una necesidad concreta. En este mismo orden, Alegría (2001) revela que la innovación tecnológica es una actividad fundamentalmente empresarial de mucha imaginación, rompe con las formas establecidas de hacer las cosas, utiliza los conocimientos disponibles y requiere la interacción con otros sectores, para la introducción comercial de nuevos productos y/o procesos al mercado y con ellos crea nuevas capacidades.

Algunos gerentes visualizan la innovación tecnológica como algo donde solo las grandes empresas pueden hacer; sin embargo, las pequeñas empresas pueden también innovar, la investigación a nivel mundial indica que las empresas innovadoras son un promedio dos veces más rentables en aquellas que no 
lo son esto es debido al grado de inversión, mientras más inversión mayor es la posición dentro del mercado. Para Escorsa (2003), la innovación tecnológica es considerada como una idea trasformada en algo vendido o usado de forma análoga, por otro lado, afirma que la innovación es el proceso en el cual a partir de una idea, invención o reconocimiento de una necesidad se desarrolló un producto, técnica o servicio útil hasta que sea comercialmente aceptado.

Otra definición fue la de Pavón y Goodman (1996), la cual la entiende como el conjunto de actividades inscritas en un determinado período de tiempo y lugar que conducen a la introducción con éxito en el mercado, por primera vez, de una idea en forma de nuevos o mejores productos, servicios o técnicos de gestión y organización.

De lo anterior, se puede referir en lo siguiente, la innovación tecnológica requiere de un entorno adecuado en donde intervienen factores como; disponibilidad de personal calificado, centros de formación e investigación, financiamiento, interrelación proveedor usuario, información tecnológica y de mercado, canales de distribución, compras públicas, empresas auxiliares, comunicación e infraestructura. Es decir, se entiende la aplicación de una innovación cuando se ha puesto en el mercado o se ha utilizado en un proceso de producción. En este sentido la innovación tecnológica ya sea de producto o de proceso, en las empresas adquiere características peculiares, está sujeta a restricciones asociadas a la base natural de su producción y características del consumo. Por otro lado, la innovación tecnológica va más allá de la investigación y desarrollo, en la medida en que se comprenden todas las fases científicas técnicas comerciales y financieras necesarias para el éxito de los productos nuevos o mejorados en sus características, o la introducción de un nuevo servicio.

\section{3.- MÉTODO}

\section{$\checkmark$ Diseño}

A continuación se expone la metodología empleada para la investigación se empelo el paradigma positivista, según Hernández, Fernández y Batista (2006), se tipificó como de carácter descriptivo, Hurtado (2010), pues su propósito fue especificar las descripción precisa del evento de estudio. Este tipo de investigación se asocia al diagnóstico, y se expone el evento estudiado, haciendo una enumeración detallada de sus características, de modo tal que en los resultados se pueden obtener dos niveles, dependiendo del fenómeno y del propósito del investigador; un nivel elemental donde se clasifica la información, y el nivel más sofisticado en el cual se ponen en relación los elementos observados a fin de obtener una descripción más detallada.

El diseño de esta investigación es no experimental de campo, transeccional o transversal descriptivo, por cuanto para operacionalizar los objetivos específicos planteados, describir la variables, analizar e 
interpretar las informaciones que se registren es fundamental acudir a la realidad y contexto objeto de estudio, es decir, el sitio donde ocurren los hechos estudiados, que para el caso corresponde a la industria petrolera venezolana específicamente en la Gerencia de AIT de las empresas mixtas.

\section{$\checkmark$ Participantes}

Cabe destacar que la población para la investigación se clasificó como finita y accesible; según Chávez (2007), el señala que el número de unidades, está constituida por menos de cien mil unidades (100.000) y accesibles por su función, cabe destacar, que los investigadores tomaron toda la población para llevar a cabo el estudio, por lo que se consideró censo poblacional, según Tamayo y Tamayo (2001) siendo cincuenta y dos (52) personas, quienes se denominan informantes claves.

Basado en lo anterior, para determinar la muestra de este estudio, se planteó en principio la necesidad de fijar las características que calificaran a las unidades de observación como participantes, a partir de las cuales, se pudieran obtener los datos. (Ver tabla 1)

Tabla 1. Distribución de los informantes clave

\begin{tabular}{|c|c|c|c|c|c|c|c|c|c|c|}
\hline Empresa Mixta & $\begin{array}{c}\text { Gerente } \\
\text { AIT }\end{array}$ & Sup. AIT & $\begin{array}{c}\text { Ing. } \\
\text { Desarrolladores }\end{array}$ & $\begin{array}{c}\text { Ing. } \\
\text { Sistemas }\end{array}$ & $\begin{array}{c}\text { Ing. } \\
\text { Computación }\end{array}$ & $\begin{array}{c}\text { Analista } \\
\text { Gestión } \\
\text { del } \\
\text { Cambio }\end{array}$ & $\begin{array}{c}\text { Analista } \\
\text { Gestión de } \\
\text { Necesidades y } \\
\text { Oportunidades }\end{array}$ & $\begin{array}{c}\text { Analista TIC } \\
\text { para el } \\
\text { desarrollo e } \\
\text { Implantación de } \\
\text { Soluciones }\end{array}$ & $\begin{array}{c}\text { Analista } \\
\text { TIC para } \\
\text { el } \\
\text { Usuario }\end{array}$ & $\begin{array}{c}\text { Total } \\
\text { por } \\
\text { EM }\end{array}$ \\
\hline Petrowayu, S.A & 1 & & 4 & & & & & & & 5 \\
\hline Petrowarao, S,A & 1 & & & 3 & & & & & & 4 \\
\hline Petroquiriquire, S.A & 1 & & & & 2 & & & & & 3 \\
\hline Lagopetrol, S.A & 1 & & 2 & & & & & & & 3 \\
\hline Petroboscan, S.A & 1 & & & & & 4 & & & & 5 \\
\hline Petrocabimas, S.A & 1 & & & & & & & 2 & & 3 \\
\hline Petroindependiente, S.A & & 1 & & & & & 3 & & & 4 \\
\hline Petrolera Sinovenezolana, S.A & 1 & & & 1 & & 1 & & & 1 & 4 \\
\hline Petrolera Bielovenezolana, S.A & & 1 & 3 & & & & & & & 4 \\
\hline Baripetrol, S.A & & 1 & & 3 & & & & & & 4 \\
\hline Petroperijá, S.A & 1 & & & 4 & & & & & & 5 \\
\hline Petrocumarebo, S.A & 1 & & & & & & & 3 & & 4 \\
\hline Petroregional del Lago, S.A & 1 & & 3 & & & & & & & 4 \\
\hline TOTAL & 10 & 3 & 12 & 11 & 2 & 5 & 3 & 5 & 1 & 52 \\
\hline
\end{tabular}

Fuente: Elaboración propia (2015)

\section{$\checkmark$ Instrumento}

Se aplicó como técnica de investigación la encuesta, empleándose un instrumento tipo cuestionario auto administrado, estructurado, con preguntas, cerradas, el cual permitirá registrar la información 
requerida para luego ser procesada y de esta manera establecer las conclusiones y recomendaciones pertinentes, así como, se diseñaran formatos de análisis.

Las características de los instrumentos utilizados para el registro de los datos a partir del cuestionario, estuvo compuesto por ítems redactados de forma afirmativa y estructurado de carácter cerrado y de construcción de escala tipo Likert, “” (Hernández y col. 2006), distribuidos en veinticuatro (24) ítems para la variable políticas públicas en innovación tecnológica.

De acuerdo a esta escala, el instrumento ofrecerá cinco (05) alternativas de respuesta: siempre, casi siempre, rara vez, muy pocas veces, nunca, correspondiéndole a cada respuesta un puntaje desde cinco (05) puntos hasta un (01) punto, según el orden de presentación de alternativas indicado

De acuerdo a esta escala, el instrumento ofrece cinco (05) alternativas de respuesta: siempre, casi siempre, rara vez, muy pocas veces, nunca, correspondiéndole a cada respuesta un puntaje desde cinco (05) puntos hasta un (01) punto, según el orden de presentación de alternativas indicado, tal y como puede observarse en el cuadro 2, a continuación:

Cuadro 2. Ponderación de los ítems

\begin{tabular}{|cc|}
\hline ALTERNATIVAS & PUNTOS \\
SIEMPRE & 5 \\
CASI SIEMPRE & 4 \\
RARA VEZ & 3 \\
MUY POCAS VECES & 2 \\
NUNCA & 1 \\
\hline
\end{tabular}

Fuente: Elaboración propia (2015)

Para llevar a cabo los objetivos expuestos en esta investigación, se procedió a obtener la validez de contenido, considerada por los autores, como la verificación del dominio de las áreas específicas del trabajo de investigación a través de la opinión de los jueces expertos en relación a la estructuración del instrumento, quienes expresaron sus opiniones objetivas de cada uno de los ítems. Una vez obtenidos los resultados provenientes del análisis de los expertos, estos establecen que los instrumentos permiten alcanzar los objetivos específicos propuestos en la investigación; por responder a los indicadores, dimensiones y variables de la presente investigación.

Seguidamente, los instrumentos fueron sometidos a la validez de constructo-discriminatorio según Hernández y otros, (2006, p.238), se refiere "al grado en que una medición se relaciona consistentemente con otras mediciones de acuerdo con hipótesis derivadas teóricamente y que concierne a los conceptos (o constructo) que están siendo medidos“

Para tal propósito se aplicó una prueba piloto con 10 sujetos, con una población similar a la población objeto de estudio aplicada en las empresas mixtas del sector petrolero del occidente venezolano, Los resultados de esta prueba piloto, por medio del uso del software Microsoft Excel, fueron tabulados y 
ordenados inicialmente en grupos altos y bajos, calculado la media y varianza de cada ítems. Se utilizan los datos de la prueba piloto y la fórmula de Alpha Cronbach, la cual se aplica para estimar la confiabilidad en cuestionarios con alternativas tipo escala:

$$
r_{t t}=\left(\frac{N}{N-1}\right) \times\left(1-\frac{\sum s^{2}{ }_{i}}{s^{2} t}\right)
$$

Donde:

$$
\begin{aligned}
& r_{t t}: \text { Coeficiente de Cronbach } \\
& N \quad: \text { Número total de ítems del cuestionario } \\
& \sum s^{2}{ }_{i}: \text { Sumatoria de las varianzas de cada ítem } \\
& s^{2} t: \text { Varianza de puntuaciones totales }
\end{aligned}
$$

Una vez obtenido el resultado se verificó la magnitud de su confiabilidad, de cual se muestra en el cuadro 3.

Cuadro 3. Escala de Interpretación para los Coeficientes de Confiabilidad

\begin{tabular}{|cc|}
\hline Rango & Interpretación \\
$0,81-1,00$ & Muy Alta \\
$0,61-0.80$ & Alta \\
$0,41-0,60$ & Moderada \\
$0,21-0,40$ & Baja \\
$0,01-0,20$ & Muy Baja \\
\hline
\end{tabular}

Fuente: Elaboración propia (2015)

Una vez realizado el cálculo del coeficiente de Alfa de Cronbach, el mismo dio como resultado 0.86 para la variable, lo que indica de acuerdo al Baremo de la escala de Interpretación para los coeficientes de confiabilidad están en el rango de Muy Alta, interpretando que el instrumento es confiable para su aplicación.

La interpretación de la información obtenida a partir del formato de la encuesta, es objeto de estadísticas descriptivas, considerando la naturaleza cuantitativa de los datos y la necesidad de llevar a cabo el cálculo de frecuencias absolutas, frecuencias relativas, medidas de tendencia central y de dispersión a partir de las respuestas registradas por los individuos objeto de estudio.

Para la interpretación de los datos estadísticos, se elaboraron tablas de frecuencias y porcentajes se utilizaron baremos de interpretación, elaborados en función de los ítems que conforman cada indicador, dimensión además la variable. Estratégicamente se diseñó un arreglo único para determinar el baremo de aplicación en función del número de ítems, el cual considera las puntuaciones extremos posibles, sin posibilidad de solapamiento 
A partir de la información recabada por el instrumento, se realizó un análisis en base a parámetros propios de la estadística descriptiva como las medidas de tendencia central, media.

Cuadro 4. Baremo interpretación de las medias

\begin{tabular}{|cc|}
\hline INTERVALOS & INTERPRETACIÓN \\
Entre 1 y 1,80 & Muy Bajo Nivel \\
Entre 1,81 y 2,6 & Bajo Nivel \\
Entre 2,61 y 3,4 & Moderado Nivel \\
Entre 3,41 y 4,2 & Alto Nivel \\
Entre 4,21 y 5 & Muy alto Nivel \\
\hline
\end{tabular}

Fuente: Elaboración Propia (2015)

\section{4.- RESULTADOS}

Luego de desarrollar el análisis estadístico sobre los datos obtenidos a través del instrumento diseñado para tal fin, el cual se puede observar en el anexo A, se procedió a realizar el análisis del mismo de acuerdo a la variable planteada, las dimensiones y los indicadores que lo componen. Posteriormente, se elaboró una comparación de estos resultados con las bases teóricas y otras posiciones investigadas, donde se presentaron los resultados obtenidos a continuación:

Tabla 2. Acciones de las políticas públicas de innovación

\begin{tabular}{|c|c|c|c|c|c|c|c|}
\hline \multirow{2}{*}{ Indicadores } & \multicolumn{5}{|c|}{ Alternativas (\%) } & \multirow[b]{2}{*}{ Total } & \multirow[b]{2}{*}{$\begin{array}{c}\text { Media } \\
\text { Ponderada }\end{array}$} \\
\hline & Siempre & $\begin{array}{c}\text { Casi } \\
\text { siempre }\end{array}$ & $\begin{array}{l}\text { Algunas } \\
\text { veces }\end{array}$ & $\begin{array}{c}\text { Casi } \\
\text { nunca }\end{array}$ & Nunca & & \\
\hline Estratégicas & $27 \%$ & $35 \%$ & $13 \%$ & $10 \%$ & $15 \%$ & $100 \%$ & 3,48 \\
\hline Tácticas & $23 \%$ & $37 \%$ & $15 \%$ & $12 \%$ & $13 \%$ & $100 \%$ & 3,44 \\
\hline Rendición de Cuentas & $21 \%$ & $38 \%$ & $19 \%$ & $17 \%$ & $4 \%$ & $100 \%$ & 3,56 \\
\hline Promedio & $24 \%$ & $37 \%$ & $16 \%$ & $13 \%$ & $11 \%$ & $100 \%$ & 3,49 \\
\hline Media General & \multicolumn{7}{|c|}{3,49} \\
\hline Categoría & \multicolumn{7}{|c|}{ Alto Nivel } \\
\hline
\end{tabular}

Fuente: Elaboración propia (2015)

Para la dimensión acciones de las políticas públicas de innovación el indicador estratégicas el 27 \% de los informantes claves respondieron al instrumentos de recolección de datos a la escala de respuesta siempre, un35\% para casi siempre, un $13 \%$ para algunas veces, un $10 \%$ para casi nunca y $15 \%$ se ubicó en la escala de nunca. Arrojando una media ponderada de 3.48 que de acuerdo al baremo se ubicó en alto nivel. Para el indicador tácticas las respuestas de los informantes claves se ubicaron en $23 \%$ para siempre, un $37 \%$ para casi siempre, un $15 \%$ algunas veces, un $12 \%$ y un $8 \%$ para casi nunca y nunca respectivamente, arrojando una media ponderada de 3.44 que de acuerdo al baremo se ubicó en alto nivel.

Para el indicador rendición de cuentas las respuestas de los informantes claves se ubicaron en $24 \%$ para siempre, un $37 \%$ para casi siempre, un $19 \%$ algunas veces, un $17 \%$ y un $4 \%$ para casi nunca y 
nunca respectivamente, arrojando una media ponderada de 3.56 que de acuerdo al baremo se ubicó en alto nivel. Es importante destacar que la media de la dimensión se ubicó en 3.49 que de acuerdo al baremo se ubicó en alto nivel.

Tabla 3. Elementos de las políticas públicas de innovación

\begin{tabular}{|llllllll|}
\hline Indicadores & \multicolumn{2}{l}{ Alternativas (\%) } & & & & Media \\
& Siempre & $\begin{array}{l}\text { Casi } \\
\text { siempre }\end{array}$ & $\begin{array}{l}\text { Algunas } \\
\text { veces }\end{array}$ & $\begin{array}{l}\text { Casi } \\
\text { nunca }\end{array}$ & Nunca & $\begin{array}{l}\text { Total } \\
\text { Ponder } \\
\text { ada }\end{array}$ \\
Contexto Externo & $35 \%$ & $38 \%$ & $13 \%$ & $10 \%$ & $4 \%$ & $100 \%$ & $\mathbf{3 , 9 0}$ \\
Estrategias & $29 \%$ & $35 \%$ & $23 \%$ & $8 \%$ & $6 \%$ & $100 \%$ & $\mathbf{3 , 7 3}$ \\
Comunidades de Aprendizaje & $25 \%$ & $19 \%$ & $33 \%$ & $13 \%$ & $10 \%$ & $100 \%$ & $\mathbf{3 , 3 7}$ \\
Toma de Decisiones & $23 \%$ & $15 \%$ & $29 \%$ & $17 \%$ & $15 \%$ & $100 \%$ & $\mathbf{3 , 1 3}$ \\
Tecnología Organizativa & $21 \%$ & $31 \%$ & $27 \%$ & $6 \%$ & $15 \%$ & $100 \%$ & $\mathbf{3 , 3 7}$ \\
Arquitectura Organizacional & $23 \%$ & $21 \%$ & $29 \%$ & $12 \%$ & $15 \%$ & $100 \%$ & $\mathbf{3 , 2 5}$ \\
Promedio & $26 \%$ & $27 \%$ & $26 \%$ & $11 \%$ & $11 \%$ & $100 \%$ & $\mathbf{3 , 4 6}$ \\
Media General & $\mathbf{3 , 4 6}$ & & & & & & \\
Categoría & $\mathbf{A l t o}$ Nivel & & & & & & \\
\hline
\end{tabular}

Fuente: Elaboración propia (2015)

Para la dimensión elementos de las políticas públicas de innovación el indicador contexto externo el 35 $\%$ de los informantes claves respondieron al instrumentos de recolección de datos a la escala de respuesta siempre, un $38 \%$ para casi siempre, un $13 \%$ para algunas veces, un $10 \%$ para casi nunca y $4 \%$ se ubicó en la escala de nunca. Arrojando una media ponderada de 3.90 que de acuerdo al baremo se ubicó en alto nivel.

Para el indicador estrategias las respuestas de los informantes claves se ubicaron en $29 \%$ para siempre, un $35 \%$ para casi siempre, un $23 \%$ algunas veces, un $8 \%$ y un $6 \%$ para casi nunca y nunca respectivamente, arrojando una media ponderada de 3.73 que de acuerdo al baremo se ubicó en alto nivel. Para el indicador comunidades de aprendizaje las respuestas de los informantes claves se ubicaron en 25 $\%$ para siempre, un $19 \%$ para casi siempre, un $33 \%$ algunas veces, un $13 \%$ y un $8 \%$ para casi nunca y nunca respectivamente, arrojando una media ponderada de 3.37 que de acuerdo al baremo se ubicó en moderado nivel.

Para el indicador toma de decisiones las respuestas de los informantes claves se ubicaron en $23 \%$ para siempre, un $15 \%$ para casi siempre, un $29 \%$ algunas veces, un $17 \%$ y un $15 \%$ para casi nunca y nunca respectivamente, arrojando una media ponderada de 3.13 que de acuerdo al baremo se ubicó en moderado nivel. Para el indicador tecnología organizacional las respuestas de los informantes claves se ubicaron en $21 \%$ para siempre, un $31 \%$ para casi siempre, un $27 \%$ algunas veces, un $6 \%$ y un $15 \%$ para casi nunca y nunca respectivamente, arrojando una media ponderada de 3. 37 que de acuerdo al baremo se ubicó en moderado nivel. 
Para el indicador arquitectura organizacional las respuestas de los informantes claves se ubicaron en $23 \%$ para siempre, un $21 \%$ para casi siempre, un $29 \%$ algunas veces, un $12 \%$ y un $15 \%$ para casi nunca y nunca respectivamente, arrojando una media ponderada de 3.25 que de acuerdo al baremo se ubicó en moderado nivel. Es importante destacar que la media de la dimensión se ubicó en 3.46 que de acuerdo al baremo se ubicó en alto nivel.

\section{5.- DISCUSIÓN}

Para la dimensión acciones de las políticas públicas de innovación es importante destacar que la media de la dimensión se ubicó en 3.49 que de acuerdo al baremo se ubicó en alto nivel. Estos resultados entran en correspondencia con el autor Kingdon (2006), cuando indica que una política pública se forma o se concreta cuando tres elementos confluyen se detectan problemas, se tiene a la vista propuestas de políticas públicas alternativas y la decisión de la autoridad política. Eso viene junto, probablemente cuando una ventana de oportunidad se abre para que una propuesta sea promovida o un problema sea resuelto.

En el mismo orden de ideas, el citado autor expresa lo que hace posible un cambio en las políticas públicas es la confluencia de factores como el reconocimiento de problemas, la propuesta alternativa disponible y la definición en las circunstancias políticas. Ese momento de concurrencia es cuando se abre una ventana de oportunidad, el cual permite reducir las restricciones al rediseño de las propuestas alternativas.

Una forma de concebir la formación de las políticas públicas, como lo propone es la confluencia de una ventana de oportunidad que se abre, en virtud de un momento político o un problema social, junto con la especificación de una alternativa y la decisión de la autoridad, para luego verificar la implementación de dicha decisión.

Para la dimensión elementos de las políticas públicas de innovación la media se ubicó en 3.46 que de acuerdo al baremo se ubicó en alto nivel. Pavón e Hidalgo (2004), en su teoría afirman que algunos componentes a considerar en el proceso de innovación tales como; contexto externo, estrategia, aprendizaje, toma de decisiones, tecnología organizativa, así como arquitectura organizacional e innovación. Al respecto señala el autor que estos elementos contribuyen al gerente en donde enfocar sus esfuerzos. En consecuencia el contexto externo en el cual se identifican las oportunidades para nuevas ideas, nuevos productos o servicios. En el tejido de un proceso corporativo de innovación siendo uno de los primeros elementos a considerar. Su estudio es clave para el impacto en la organización.

A tal razón, una mirada a la dinámica del contexto externo en que se desenvuelven las organizaciones, hace referencia a la presencia básica de dos tendencias: La globalización y la aceleración por lo que se corresponden con los resultados obtenidos 
Es importante mencionar a Regnosco (2006), quien señala que a partir de estas dos tendencias aparecen mecanismos que ejercen aún más presión e impacto en la fuerza laboral, entre ellos, se encuentran una mayor competitividad en el mercado por la captación de clientes, énfasis en la gerencia de los costos, en ajustarse como en dirigir procesos de cambio, impacto de la tecnología de información en el trabajo y en las telecomunicaciones, así como en la gerencia de conocimiento.

Esta acumulación de procesos además de eventos del entorno que simultáneamente interactúan e impactan en las estrategias igualmente en las operaciones de las organizaciones, conlleva a considerar como requisito básico, identificarlos, asirlos, analizarlos, interpretarlos convirtiéndolos en conocimientos que iluminen la toma de decisiones.

En general no está referido a lo que debería ser la política como tipo ideal o conducta deseada; esa área dentro de la teoría política es característica de la filosofía política. Tampoco es el estudio de los elementos formales de la política como lo son las leyes, su formación y las intenciones de éstas, tarea de la jurisprudencia. La ciencia política tiene en cuenta el comportamiento político efectivo y observable de las personas y las sociedades.

Sin embargo, el estado venezolano presenta una robusta normativa y regulaciones legales en políticas públicas de innovación que están orientadas en el manejo de los procesos tecnológicos en la industria petrolera venezolana, así como se pudo identificar las acciones que generan las políticas públicas en innovación en la industria petrolera venezolana de acuerdo al siguiente orden: rendición de cuentas, estrategias y por último las tácticas, se recomienda identificar las acciones que generan las políticas públicas en innovación tecnológica en la industria petrolera venezolana para potenciar las tácticas en las acciones como políticas públicas de innovación.

De igual forma se establecieron los elementos de la innovación que apoyan las políticas públicas de innovación en la industria petrolera, se establecieron de acuerdo a los resultados obteniéndose en primer lugar el contexto externo, estrategias, comunidades de aprendizaje, tecnología organizacional, arquitectura organizacional y toma de decisiones, así mismo se recomienda se deben hacer reuniones para generar tormenta de ideas que promuevan un clima favorable para la toma de decisiones.

Se presentan a continuación las conclusiones del estudio en cuanto a identificar las acciones que generan las políticas públicas en innovación tecnológica en la industria petrolera venezolana se identificaron cada una de las acciones de acuerdo al siguiente orden rendición de cuentas, estrategias y por último las tácticas.

Así mismo se establecieron los elementos de la innovación que apoyan las políticas públicas de innovación tecnológica en la industria petrolera, se establecieron de acuerdo a los resultados obteniéndose 
en primer lugar el contexto externo, estrategias, comunidades de aprendizaje, tecnología organizacional, arquitectura organizacional y toma de decisiones.

\section{6.- REFERENCIAS}

Alcántara, M. (1995). Gobernabilidad, crisis y cambios. Fondo de cultura económica. México.

Arias, F. (2006). El Proyecto de Investigación. Introducción a la metodología científica. (5ª ed.). Editorial Episteme. Caracas, Venezuela.

Bourg, D. (2005). ¿Cuál es el futuro del desarrollo sostenible?. Ediciones Akal, S.A. Madrid - España.

Carballo, R. (2004). En La Espiral de la Innovación. Ediciones Díaz de Santos, S.A. Madrid, España.

Cebrián, J. A., López, J.M. y Serra, P. (2003). Urbanización y Globalización, Revista Cooperación Internacional, n. 9, pp. 63-76.

Chávez, N. (2007). Introducción a la Investigación Educativa. Taller de Artes Gráficas, S.A. Maracaibo, Venezuela.

Claros, O. (2000). Control Fiscal un instrumento para el desarrollo. ECOE Ediciones, Bogotá, Colombia. Constitución de la República Bolivariana de Venezuela. Gaceta Oficial extraordinaria No. 5.453 del 24 de marzo del 2000. Editorial Graficas 2021 c.a.

COTEC (2001). Innovación Tecnológica. Ideas Básicas. Fundación para la Innovación Tecnológica, España. [Documento en línea]. Disponible en http://www.cotec.es.

Damiani, Luis. (2005). Epistemología y ciencia en la modernidad. FACES. UCV. Caracas. P.p 27- 30.

Escorsa, Pere \& Valls, Jaume (2003). Tecnología e innovación en la empresa. (2a. Ed.) Ediciones de la Universidad Politécnica de Cataluña, SL, Barcelona, España.

Genatios, Carlos (2004) La ciencia y la tecnología en la construcción del futuro del país. Ciclo de Foros Nacionales. Caracas. Publicaciones MCT.

Enríquez, L. A., Salgado, M. C., \& Castorena, O. H. (2013). La influencia de las tecnologías de la información en los procesos productivos para una mayor competitividad de la pyme de aguas calientes: un estudio empírico. En Revista Desarrollo Gerencial, 5 (1) Pp.40-68- Universidad Simón Bolivvar - Colombia

Hurtado de Barrera, Jacqueline. (2010) El proyecto de investigación: metodología de la investigación. Edición: 2a. ed. Descripción: 119 p.: il.; 21 cm.ISBN: 9806306201 . Caracas 
Kuhn, T. (2005). De los Paradigmas a la teoría Evolucionista. Editorial Universitaria de Buenos aires. Buenos Aires, Argentina.

Martínez, J. C. (2002). La innovación tecnológica en las sociedades cooperativas y otras organizaciones de participación. Revesco. Revista de Estudios Cooperativos, (78), 7-25.

Ministerio de industrias ligeras y pro competencia, (2006), Superintendencia para la promoción y protección de la libre competencia www.procompetencia.gov.ve/pptransportemercancia.html. Venezuela, [Consulta: mayo 08, 2014].

Nohlen, D., (2006). Diccionario de Ciencia Política. Teorías, métodos, conceptos. Porrúa, Ciudad de México 2006, ISBN 970-07-6115-0

Nueva York.

Oberto, A (2007). Conocimiento tecnológico e innovación en el sector de termoplásticos del estado Zulia. Venezuela. Universidad del Zulia Ediciones del Vicerrectora Académico.

Pavón, J. y Goodman, K. (1996). Proyecto MODELTEC. La planificación del desarrollo tecnológico en el caso español. Madrid.

Pelekais, C., Finol, M., Neuman, N. y Belloso, O. (2007). El ABC de la investigación. Una aproximación teórico práctica. Ediciones Astro Data S.A. $2^{\text {a }}$ edición. Maracaibo, Venezuela.

Podestá Arzubiaga, J. (2001). Problematización de las políticas públicas desde la óptica regional. Última década, 9(15), 163-175. Valparaíso-Chile.

Prieto, R., Emonet, P., García, J., \& González, D. (2015). Cambio organizacional como estrategia de gestión en las empresas mixtas del sector petrolero. Revista de Ciencias Sociales, Vol. XXI, No.

\section{Pp. 386-402. Universidad del Zulia-Venezuela.}

PROYECTO RED PREANDINO. 2002. Documento de la Corporación Andina de Fomento. Por competencias. Editorial Débora Feely. Buenos Aires, Argentina

Romero Villafranca y Zúnica Ramajo (2005) métodos estadísticos en ingeniería. Universidad Politécnica de Valencia,

Roth, André-Noel. (2004). Políticas públicas: formulación, implementación y evaluación. Ediciones Aurora. Bogotá, DC. Primera Edición. Cuarta reimpresión.

Salazar, C. (2000). Las políticas públicas. Universidad Javeriana.

Schumpeter, J. (1942). Capitalismo, socialismo y democracia. Madrid. 
Tamayo y Tamayo, (2001) El proceso de la investigación científica. Limusa Noriega Editores. Cuarta edición.

Vallès, J. (2000). Ciencia Política. Una introducción. Barcelona, ISBN 978-84-3441-824-0

Vásquez, Miguel, (2004) Las políticas públicas y los compromisos de la investigación. Políticas públicas ambientales. 